\title{
Corrective exercises specific for adolescent idiopathic scoliosis presented with bilateral leg pain: a case report describing an adaptive program and the recorded improvement as expressed by Cobb angle
}

\author{
DOI: https://doi.org/10.5114/pq.2020.96237
}

\author{
Fatma Elzahraa AbdelAziz ElBayomi', Faten Fathy Ibrahim 1 , Mona M. Ibrahim² \\ ${ }^{1}$ Orthopaedic Outpatient Clinic, Faculty of Physical Therapy, Cairo University, Giza, Egypt \\ 2 Department of Physical Therapy for Musculoskeletal Disorders and Its Surgery, Faculty of Physical Therapy, \\ Cairo University, Giza, Egypt
}

\begin{abstract}
Adolescent idiopathic scoliosis is considered as the most common type of scoliosis. A 13-year-old female complained of bilateral leg pain, left > right, and severe headache. She was referred to an orthopaedic physical therapy clinic. Spinal X-ray revealed right thoracolumbar scoliosis with Cobb angle of about $48^{\circ}$. The primary curve was in the lumbar vertebrae, directed to the left, and the secondary curve was in the thoracic vertebrae, directed to the right. Two surgeons recommended corrective surgery, while one recommended physiotherapy for 6 months. The third surgical opinion was based on magnetic resonance imaging that presented normal cervical spine, mild scoliotic deformity of lower dorsal vertebrae with right-sided convexity and of lumbar vertebrae with left-sided convexity, L4-5 broad based posterior and left posterolateral disc protrusion, and L5-S1 mild central posterior disc protrusion. At the final evaluation after 18 months of a corrective exercise program, the patient reported no symptoms at rest or with exercises. The improvement was evidenced by Cobb angle change from $48^{\circ}$ to $21^{\circ}$, pelvic shift, and general spinal flexibility and strength. The current case report describes a specific corrective exercise protocol adapted to a case of adolescent idiopathic scoliosis that presented with bilateral leg pain.
\end{abstract}

Key words: adolescent idiopathic scoliosis, specific exercise, correction, physical therapy, Cobb angle

\section{Introduction}

Scoliosis is considered as a group of conditions associated with variable changes in the shape and position of the spine, thorax, and trunk [1]. It is characterized by the presence of a lateral curvature of the vertebral column in the coronal plane [2]. Scoliosis may be structural or functional. In structural scoliosis, the coronal plane curvature cannot be corrected by ipsilateral forward bending owing to the presence of a 3-dimensional deformity involving all planes. In turn, functional scoliosis becomes completely corrected upon forward trunk bending [2, 3]. The term 'idiopathic scoliosis' is used to describe structural scoliosis that is not associated with any specific disease causing spinal deformation [4]. Idiopathic scoliosis is further classified depending on the age of onset into infantile (up to 2 years), juvenile (3-9 years), and adolescent ( $\geq 10$ years). Adolescent idiopathic scoliosis (AIS) is the most common type of scoliosis, accounting for $80-85 \%$ of scoliosis cases [5].

The clinical features of AIS include asymmetry recognized by a scapular prominence and a rib hump upon forward bending. The patients frequently complain of backache and fatigue with minimal activity. The standard imaging modality used to diagnose structural scoliosis is plain X-ray. It enables an objective localization and measurement of vertebral, rib, and pelvic deformities and further assess the efficiency of any conservative or surgical intervention [2].

In rehabilitation medicine, randomized controlled trials are somehow difficult to conduct because of problems in collecting a homogenous large sample to increase the power of the study. Furthermore, it is often challenging to find a suitable placebo intervention owing to both ethical and practical reasons. Moreover, it is usually hard to differentiate between the specific effect of exercise intervention and the therapeutic effect of the relationship between the patient and the therapist, which may be modified by the therapist's experience and faith in the applied therapeutic technique. Finally, it is always impossible to perform a double-blind study in rehabilitation research. On the other hand, performing good quality studies in not impossible; in fact, the Cochrane Library contains many systematic reviews on various rehabilitative interventions, which include randomized controlled trials of acceptable quality that try to overcome the previously mentioned difficulties [6].

The use of physical therapy as a conservative treatment for AIS is probably not broadly recommended by orthopaedic surgeons. A survey was conducted to screen the attitudes of the Scoliosis Research Society members toward physical therapy or physiotherapeutic scoliosis-specific exercises (PSSE) applied in AIS. The majority of the respondents (about 263) prescribed neither physical therapy nor PSSE; their main reasons were lack of evidence and the sense that physical therapy had no value in these cases. Only $22 \%$ of the surgeons supported prescribing PSSE for AIS [7]. Corrective exercises are among the most common physical therapy interventions to treat AIS. Zapata et al. [8] used corrective exercises in cases of AIS. A year after the exercises, they found a significant improvement of Cobb angle compared with relaxed standing for the primary lumbar curve. They ascribe this observation to the improvement of spinal

Correspondence address: Mona M. Ibrahim, Department of Physical Therapy for Musculoskeletal Disorders and Its Surgery,

Faculty of Physical Therapy, Cairo University, 1 Gamaa Street, Giza, Egypt, postal code: 12613, e-mail: mona.ibrahiem@pt.cu.edu.eg

Citation: EIBayomi FEA, Ibrahim FF, Ibrahim MM. Corrective exercises specific for adolescent idiopathic scoliosis presented with bilateral leg pain: a case report describing an adaptive program and the recorded improvement as expressed by Cobb angle. Physiother Quart. 2020; 28(4):55-60; doi: https://doi.org/10.5114/pq.2020.96237. 
flexibility and mobility. On the other hand, no immediate improvement was demonstrated, which indicates that appropriate time is needed to achieve the required spinal results [8].

The purpose of the current case report is to present an effective treatment plan for a 13-year-old female patient who suffered from bilateral leg pain associated with AIS with Cobb angle of $48^{\circ}$ and sought for a chance of physical therapy for 6 months as an attempt to correct the deformity and avoid operative intervention.

The patient started physical therapy treatment in January 2017 at the Orthopaedic Physical Therapy Clinic at the Faculty of Physical Therapy, Cairo University.

\section{Ethical approval}

The research related to human use has complied with all the relevant national regulations and institutional policies, has followed the tenets of the Declaration of Helsinki, and has been approved by the authors' institutional review board or an equivalent committee.

\section{Informed consent}

The patient's informed consent has been obtained for the publication of this case report.

\section{Case description}

\section{History}

A 13-year-old female patient complained of bilateral leg pain, left > right, and severe headache. She was referred to the orthopaedic outpatient physical therapy clinic. Spinal X-ray (Figure 1) revealed right thoracolumbar scoliosis with Cobb angle of about $48^{\circ}$ in neutral position. The primary curve was in the lumbar vertebrae, directed to the left side, and the second curve was in the thoracic vertebrae, directed to the right side. Two surgeons recommended corrective surgery, while one recommended physiotherapy for 6 months.

The third surgical opinion was based on magnetic resonance imaging which presented the following:

1. Normal cervical spine.

2. Mild scoliotic deformity of lower dorsal vertebrae with right-sided convexity and of lumbar vertebrae with left-sided convexity.



Figure 1. Spinal X-ray revealing right thoracolumbar scoliosis that extends from T2-L3 with Cobb angle of $48^{\circ}$

(26.08.2016)
3. L4-5 broad based posterior and left posterolateral disc protrusion.

4. L5-S1 mild central posterior disc protrusion.

The black signals at the X-ray represent gases in the colon.

\section{Examination (0-6 months)}

At the time of the initial examination, the patient reported bilateral knee pain and complained of cosmetic dissatisfaction due to a pelvic shift. In the initial physical examination, the following findings were noted:

1. The pelvis severely shifted to the left.

2. Leg length discrepancy of about $2 \mathrm{~cm}$ right more than left by tape measurement.

3. Anterior tilting of the right ilium with posterior tilting of the left reported by palpation of anterior superior iliac spine.

4. Tight right quadratus lumborum tested by flexibility testing from side lying on the left side with the right lower limb outside the plinth downward and end feel of tightness before complete range of motion.

5. Tight both hamstrings tested by flexibility testing from supine lying with passive straight leg raising and end feel of tightness before complete range of motion.

\section{Intervention (0-6 months)}

In the first 6 months, the treatment program was conducted at a frequency of 3 sessions per week. It consisted of therapeutic exercises, manual therapy, and proprioceptive training. The following items were implemented:

1. Advising the patient to use a foot insole to compensate for the leg length discrepancy.

2. Infrared at the lower back for 15 minutes.

3. Stretching exercise for the right quadratus lumborum while the patient at her left side, upper chest rotated toward the plinth, left lower limb flexed more than $90^{\circ}$ in the hip and knee, and the right lower limb extended outside the plinth. The therapist grasped at the iliac crest and pulled downward.

4. Stretching exercise for the left trunk muscles including paraspinals, quadratus lumborum, and latissimus dorsi. The patient lay on her right side at the edge of the bed, placing roll at her lower ribs, while the therapist was fixating her lower limbs; the patient's upper limbs were outside the plinth, allowing the gravity to stretch the muscles. The therapist maintained the alignment and applied overstretch at the lower ribs. The position was maintained for 20 seconds and repeated 3 times.

5. Bilateral stretching for the hamstring muscles while the patient supine lying and straight leg raise start with one lower limb, then stretching the other, maintaining, stretching for 20 seconds and repeating 3 times.

6. General spinal manipulation for right facets at lumbar vertebrae. The patient was at her left side with hips at more than $90^{\circ}$ and uppermost knee flexed over the extended lowermost one. The therapist rotated the chest and upper body to make the patient face the ceiling and applied a high-velocity low-amplitude thrust.

7. Mobilization of right ilium posteriorly and left ilium anteriorly.

8. Pelvic shift mobilization toward the right side. The therapist stood at the right side of the patient and asked the patient to put her right arm at the therapist's shoulder, grasped the pelvis of the patient with both hands, and then moved the pelvis from left to right.

9. Abdominal strengthening exercises started gradually from $2-3$ sets, 10 repetitions. The patient lay supine, flexed 
one knee, and extended the other to increase the core muscles firing. The patient curled up till clearance of the scapulae while maintaining normal breathing.

10. Bridging exercises started gradually up to 3 sets, 10 repetitions each, with 5 seconds hold. The patient was in crook lying position, sucked her abdomen, contracted glutei, then elevated her pelvis, maintained for 5 seconds, and relaxed.

11. After about 4 months, we started to make mobilization with movement for thoracic rotation with the patient sitting on a ball to balance her pelvis (for lower thoracic spine T10-11).

12. Anteroposterior balance training on a balance board, anteroposterior direction, in front of a mirror for 5 minutes (after 4 months).

During the first 6 months of treatment with this exercise program, the patient complained of leg pain only, but at the end of these 6 months, the leg pain improved by $60 \%$ as expressed by the patient. At the end of the first 6 months, $\mathrm{X}$-ray was performed to record any changes reflected in Cobb angle. The angle measured about $35^{\circ}$, with about $13^{\circ}$ reduction from the previous record. Furthermore, the pelvic shift was improved, as seen in Figure 2.

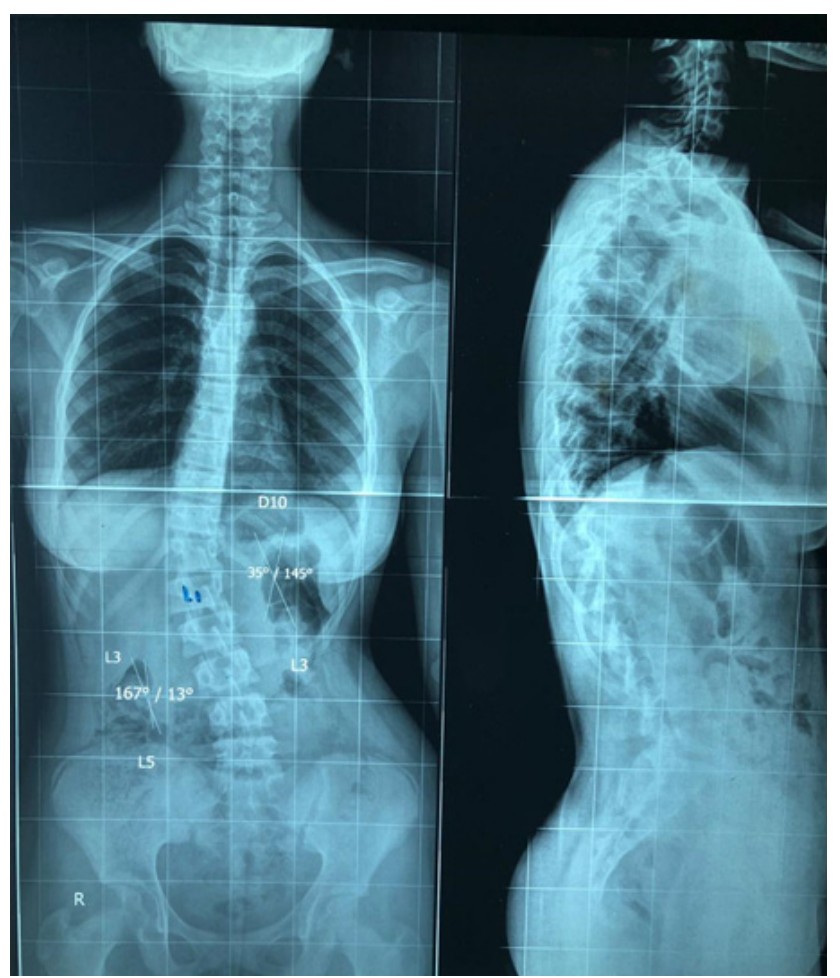

Figure 2. X-ray revealing $35^{\circ} \mathrm{Cobb}$ angle with improvement in pelvic shift

\section{Examination (6-12 months)}

The second clinical examination, performed after 6 months, revealed improvements in the following:

1. Pelvic shift, noted at X-ray and with inspection.

2. Flexibility of the right quadratus lumborum (with the same stretching position).

3. Flexibility of both hamstrings (passive straight leg raise).

4. General body balance (the patient was able to maintain her balance at a balance board in the anteroposterior direction for 5 minutes).

\section{Intervention (6-12 months)}

Thoracic rotation revealed during the second clinical assessment represented the main target for correction in the next 6 months (6-12 months) with a frequency of 2 sessions per week. The treatment program used in the next 6 months included the following:

1. Core training exercises in the form of:

a. Abdominal exercises ( 3 sets, 10 repetitions each).

b. Bridging exercises ( 3 sets, 10 repetitions each, with 5-second hold).

c. Half-bridging exercise ( 1 set, 10 repetitions on each side) with the patient in crook lying position with one lower limb flexed and the other extended. The patient was advised to contract lower abdomen and glutei, then raise her pelvis and extend the lower limb.

d. Plank exercise (1 set, 10 repetitions, with 5-second hold). The patient lay prone on her elbows and toes, then elevated her body weight on 4 points: both elbows and tip toes, maintaining the back straight for 5 seconds.

e. Side plank exercises ( 1 set, 10 repetitions, with 5-second hold on each side). The patient was side lying, flexed her knees to $90^{\circ}$, maintained the whole body in one line, supported her body at her elbow and knee while raising the pelvis.

2. Bilateral stretching exercises for hamstring muscles to maintain their flexibility.

3. Manipulation bilaterally at lumbar vertebrae to maintain lumbar mobility.

4. Posterior manipulation at the right ilium. From supine lying, the therapist passively elevated the patient's right lower limb up to $45^{\circ}$, with hip flexion and full internal rotation to lock the hip joint, and provided a low-amplitude pulling thrust and anterior manipulation at the left ilium. With the patient in supine lying position, the therapist grasped just above the ankle, elevated the left lower limb till $10^{\circ}$, and internally rotated the hip, then applied a high-velocity lowamplitude thrust.

5. While sitting on ball, bilateral shoulder extension exercise with the use of an elastic band ( 3 sets, 10 repetitions each, with 5-second hold). The patient was sitting on a Swiss ball. In front of a wall bar, she was instructed to maintain her pelvis levelled. The therapist used a medium resistance green elastic band at the wall bar above the patient's shoulder level, then the patient was instructed to bilaterally extend her upper limbs while the back was erect.

6. While sitting on a ball, thoracic rotation toward the left side with an elastic band (3 sets, 10 repetitions each). The patient was sitting on a Swiss ball, the wall bar was at her right side, and a medium resistance elastic band was at her shoulder level. The patient was asked to grasp the elastic band while her both shoulders remained at $90^{\circ}$ flexion, then rotate toward the left side against resistance.

7. Balance training on a balance board in anteroposterior and mediolateral directions for about 5 minutes in each direction in front of a mirror. The therapist stood at a side of the patient to give her instructions to maintain her balance and for the patient's safety. The exercise was graded from open eyes to closed eyes and from wide base of support to narrow base of support.

8. Mobilization for pelvic shift toward the right side.

9. Gait training exercises on a treadmill for 10 minutes to improve posture and cardiovascular fitness (speed level: from 5 to 7). The patient was instructed to maintain her back straight and give the same timing for both lower limbs.

\section{Examination (12-18 months)}

At the end of the next 6 months, another X-ray was performed, which revealed improvement in the following:

1. Cobb angle decrease to $22^{\circ}$. 


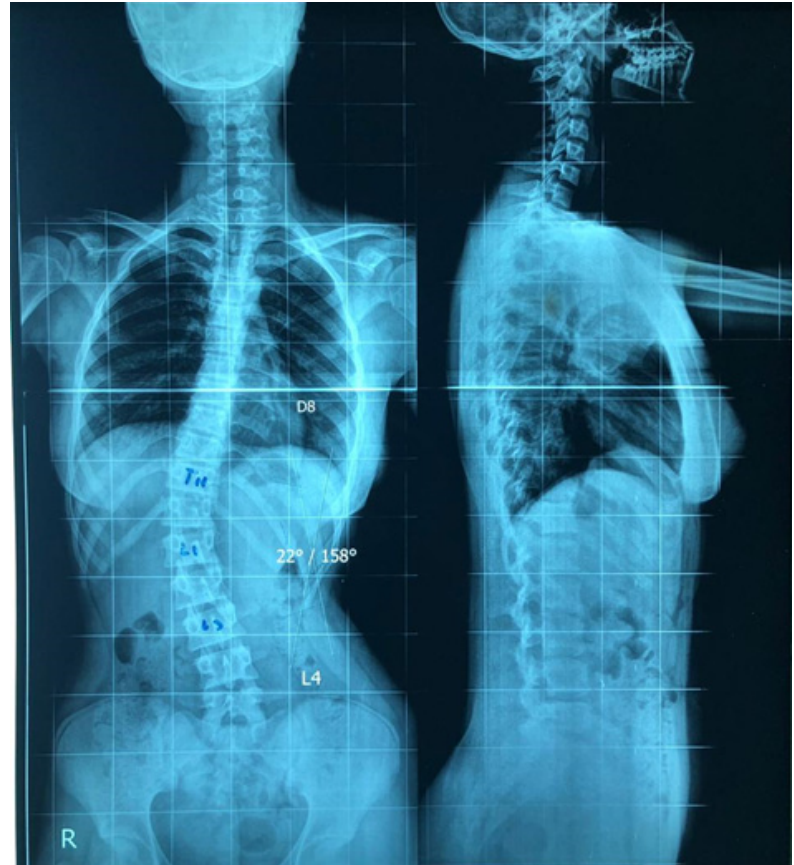

Figure 3. Improvement in Cobb angle $\left(22^{\circ}\right)$ after 12 months of the physical therapy program

2. The position of iliac bone and pelvic shift.

3. Dorsolumbar rotation (this improvement was compensated by cervical region, especially atlanto-occipital joint and head shift toward the right side, as seen in Figure 3).

4. No complaining of headache.

Intervention (12-18 months)

The treatment protocol in the next 6 months (12-18 months) was the same as in the previous 6 months, with the addition of the following:

1. Cervical spine manipulation. The patient was supine lying and her head outside the plinth, while the cervical spine at neutral position. The therapist side-bent the head of the patient to the end of range and rotated till the end-feel, then applied a high-velocity low-amplitude thrust bilaterally.

2. Manual correction of the cervical spine for head shift. The patient lay supine with the head outside the plinth. The therapist stabilized the head by one hand and the other hand grasped the cervical spine, mobilizing it from left to right.

3. Bilateral stretching for both sides of the neck. Upper trapezius and scalene stretching. The therapist side-bent the head of the patient till the spring end-feel, then maintained the stretch for 15 seconds.

4. Training at a multidirectional balance board (circle balance board) in front of a mirror, graded from a squared balance board to a circle one.

5. Gait training on a treadmill for 20 minutes, with a gradual increase to 30 minutes.

6. Mini-squat exercises with a roll behind the upper back to remain erect. The patient slid her back on the roll during the mini-squats.

7. Strengthening exercises for hip abductors and external rotators with the use of an elastic band attached above the knee and side-walking with mini-squats.

At the end of the treatment period (after 18 months), additional X-ray was performed and revealed an improvement in Cobb angle by only $1^{\circ}$ to reach $21^{\circ}$. Cervical compensation was further improved and flattening of the lumbar curve was observed, as seen in Figure 4.

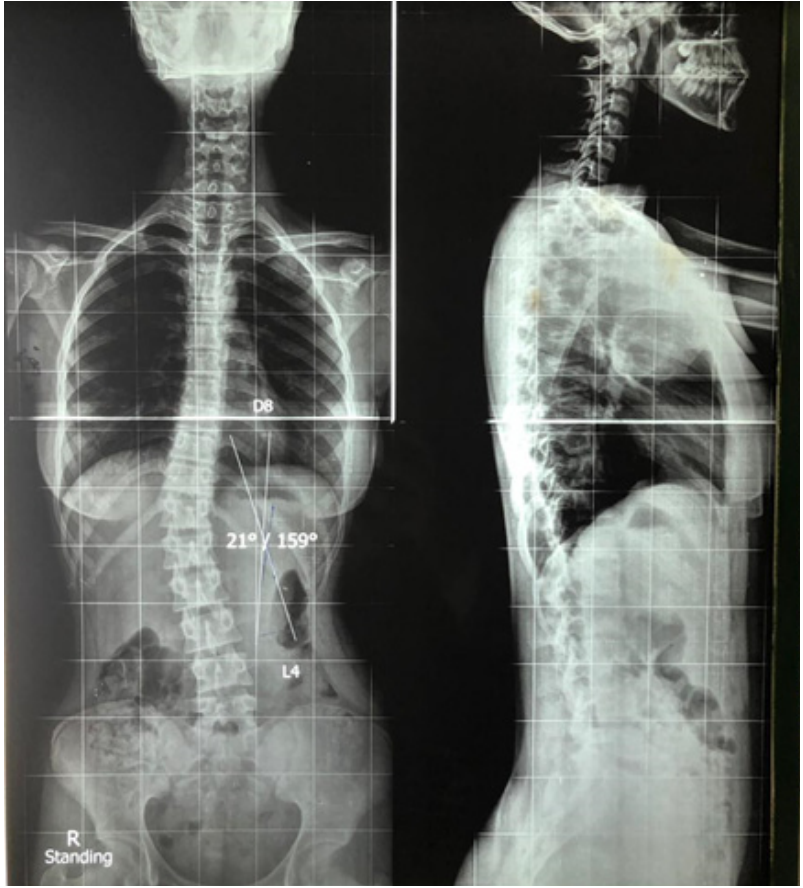

Figure 4. X-ray after 18 months of the physical therapy treatment

\section{Discussion}

The most commonly used physical therapy intervention for the treatment of AIS is manipulation with exercises. Therefore, the current approach in the case report focused on manipulation with corrective exercises and reflected a reduction in Cobb angle, monitored 3 times during the treatment. The initial Cobb angle, measured before the treatment, was $48^{\circ}$ and reduced to $21^{\circ}$ after 18 months of manipulation with exercise. The improvement was reflected in the dorsolumbar rotation, as demonstrated in routine X-ray, and pelvic shift reduction. The overall flexibility and strength of trunk and leg muscles improved. Furthermore, the active ability to correct and maintain the corrected position of the spine improved at the end of the follow-up period, which helps to preserve the reported mechanical improvements. The patient's complains of headache and poor back shapes disappeared at the second follow-up period, after 12 months. The program of treatment was individualized on the basis of the result of the initial physical examination and followed up during the total period of intervention.

A previous study showed the effectiveness of PSSE to avoid failure and reduce the need for bracing with minor Cobb angle $<25^{\circ}$. The applied exercise program improved Cobb angle by $5.3^{\circ}$ at skeletal maturity [9]. Furthermore, a supervised physical therapy program for 8 weeks in the form of spinal stabilization exercise was found effective in reducing low back pain and improving function in patients with AIS as measured by the Numeric Pain Rating Scale and the Patient-Specific Functional Scale scores, respectively [10].

Cobb angle reduction was associated with disappearance of sagittal plane curvature of the spine, as seen in Figure 4. The normal kyphotic and lordotic curves of the spine in the sagittal plane help to distribute the overall weight over the vertebral segments and increase the shock absorbing capacity of the spine. Absence of these normal curves disturbs the normal distribution of forces over the spine and lower extremities and predisposes to overuse injuries. The current observation of minimized sagittal plane curves may be ex- 
plained in the sight of scoliotic deformity. The failure of sagittal plane curvature development during growth may be reflected by a frontal plane curvature appearing in idiopathic scoliosis. Until now, there is no more than a narrative review reporting this relation of sagittal plane vertebral shape with frontal plane deformation observed in scoliosis. An emphasis was put on the biomechanics and biology of vertebral shape during growth to explore the cause of spinal scoliosis development [3]. A relation may exist between sagittal and frontal plane curves, which may explain the reason for idiopathic scoliosis; the issue needs further research with a comprehensive biomechanical follow-up since birth until maturity.

Schreiber et al. [11] studied the effect of Schroth exercise combined with a standard physical therapy program in AIS. The follow-up continued to 12 months and revealed improvements regardless changes in Cobb angle. A further work studied the combined effect of adding Schroth exercise to inside brace in cases of AIS. Schroth exercises with bracing were found more effective than bracing alone in improving Cobb angle and trunk rotation [12]. A similar result was obtained by adding Schroth exercise to standard care of patient with AIS [13]. Improvements were also observed in terms of pain scores and back muscle endurance after 3 months of Schroth exercises [14].

Direct physical therapy intervention is extremely important and found superior to prescribed home programs with minimal visits. Supervised exercise intervention turns out more effective than only one treatment in reducing pain and improves function in low back pain associated with AIS [10, 15]. The close observation and assessment during treatment help to explain the source of biomechanical faults and to correct and minimize their consequences. During the progression of treatment, the weekly visits may be minimized, and a home program is more emphasized with regular reassessment to follow curve progression, as currently used in the case report.

The benefit of exercise intervention in cases of AIS extends to prevention of curve progression in several published reports [16]. Exercises successfully stopped the progression of scoliotic curve by more than $5^{\circ}$ in Cobb angle compared with the control group without exercise intervention. The results supported the evidence of a beneficial effect of PSSE for AIS with mild curves during the period of growth before skeletal maturity [17]. These findings are in line with the growing evidence of the benefit of an early specific exercise intervention to decrease curve progression in mild $\left(<20^{\circ}\right.$ Cobb angle) growing AIS as reported by recent guidelines [1]. Further research emphasized the beneficial effect of adding orthotics to specific exercises in cases of AIS. Improvements were observed in respiratory function and back muscle endurance [18].

Reports imply that the main problem of vertebral rotation associated with AIS may be greatly associated with a muscle strength problem. The recorded myoelectric activity of muscles revealed asymmetry between both trunk sides. The asymmetry was corrected by using strengthening exercises; improvement was found in 12 female subjects with growing AIS [19]. Further reports explored the relation between AIS and the strength of trunk muscles. No rotational strength asymmetry was observed at baseline before supervised strengthening training intervention. The training program extended for 4 months proved ineffective in controlling AIS curves [20].

A recent randomized controlled trial compared the effectiveness of bracing versus exercises on Cobb angle in cases of AIS. The conclusion was that bracing and exercises showed similar effectiveness in patients with AIS. Bracing was found superior to correct the spinal deformity, while exercises were more efficient from the functional and psychological perspectives [21]. Furthermore, the choice of bracing must be individualized in accordance with the severity of deformity and patient's personality [22].

Although the literature is very rich with clinical studies exploring the effect of specific exercises on curve progression and severity, there is a lack of high-quality evidence to recommended their effectiveness for AIS patients [23]. Further high-quality studies are needed to support the efficiency of exercise intervention in those cases [22]. Nevertheless, moderate evidence comes from a recent meta-analysis on the effectiveness of exercises in reducing Cobb angle, angle of rotation, thoracic kyphosis, and lumbar lordosis. Furthermore, there is low-quality evidence that exercises reduced the average lateral deviation of the trunk. Additionally, moderate-quality evidence supports the role of exercises in improving quality of life in patients with AIS [24].

Finally, basic body awareness therapy was found extremely important for managing patients with AIS. Adding body awareness therapy to bracing improved scoliosis in terms of curve magnitude, trunk deformity, and postural deviations [25]. Core stabilization exercise was used in the current work to increase the patient's ability to actively control and correct the spinal deformity associated with AIS. Further studies supported its effectiveness in reducing rotation deformity and pain. It was proved superior to traditional exercises in conservative rehabilitation of AIS patients. Therefore, it is particularly recommended to add stabilization exercises to the current protocol of exercises for patients with AIS [26].

\section{Disclosure statement}

No author has any financial interest or received any financial benefit from this research.

\section{Conflict of interest}

The authors state no conflict of interest.

\section{References}

1. Negrini S, Donzelli S, Aulisa AG, Czaprowski D, Schreiber S, de Mauroy JC, et al. 2016 SOSORT guidelines: orthopaedic and rehabilitation treatment of idiopathic scoliosis during growth. Scoliosis Spinal Disord. 2018; 13:3; doi: 10.1186/s13013-017-0145-8.

2. Oestreich AE, Young LW, Poussaint TY. Scoliosis circa 2000: radiologic imaging perspective. Skeletal Radiol. 1998;27(11):591-605; doi: 10.1007/s002560050445.

3. Millner RA, Dickson PA. Idiopathic scoliosis: biomechanics and biology. Eur Spine J. 1996;5(6):362-373; doi: 10.1007/BF00301963.

4. Kleinberg S. The operative treatment of scoliosis. Arch Surg. 1922;5(3):631-645; doi: 10.1001/archsurg.1922. 01110150184008.

5. Eardley-Harris N, Munn Z, Cundy PJ, Gieroba TJ. The effectiveness of selective thoracic fusion for treating adolescent idiopathic scoliosis: a systematic review protocol. JBI Database System Rev Implement Rep. 2015;13(11): 4-16; doi: 10.11124/jbisrir-2015-2338.

6. Negrini S, Antonini G, Carabalona R, Minozzi S. Physical exercises as a treatment for adolescent idiopathic scoliosis. A systematic review. Pediatr Rehabil. 2003;6(3-4): 227-235; doi: 10.1080/13638490310001636781.

7. Marti CL, Glassman SD, Knott PT, Carreon LY, Hresko MT. Scoliosis Research Society members attitudes towards physical therapy and physiotherapeutic scoliosis spe- 
cific exercises for adolescent idiopathic scoliosis. Scoliosis. 2015;10:16; doi: 10.1186/s13013-015-0041-z.

8. Zapata K, Parent EC, Sucato D. Immediate effects of scoliosis-specific corrective exercises on the Cobb angle after one week and after one year of practice. Scoliosis Spinal Disord. 2016;11(Suppl. 2):36; doi: 10.1186/ s13013-016-0101-z.

9. Negrini S, Donzelli S, Negrini A, Parzini S, Romano M, Zaina F. Specific exercises reduce the need for bracing in adolescents with idiopathic scoliosis: a practical clinical trial. Ann Phys Rehabil Med. 2019;62(2):69-76; doi: 10.1016/j.rehab.2018.07.010.

10. Zapata KA, Wang-Price SS, Sucato DJ, Thompson M, Trudelle-Jackson E, Lovelace-Chandler V. Spinal stabilization exercise effectiveness for low back pain in adolescent idiopathic scoliosis: a randomized trial. Pediatr Phys Ther. 2015;27(4):396-402; doi: 10.1097/PEP. 0000000000000174.

11. Schreiber S, Parent EC, Hedden DM, Moreau M, Hill D, Lou E. Effect of Schroth exercises on curve characteristics and clinical outcomes in adolescent idiopathic scoliosis: protocol for a multicentre randomised controlled trial. J Physiother. 2014;60(4):234; doi: 10.1016/j.jphys. 2014.08.005.

12. Kwan KYH, Cheng ACS, Koh HY, Chiu AYY, Cheung KMC. Effectiveness of Schroth exercises during bracing in adolescent idiopathic scoliosis: results from a preliminary study - SOSORT Award 2017 Winner. Scoliosis Spinal Disord. 2017;12:32; doi: 10.1186/s13013-017-0139-6.

13. Schreiber S, Parent EC, Moez EK, Hedden DM, Hill DL, Moreau M, et al. Schroth physiotherapeutic scoliosisspecific exercises added to the standard of care lead to better Cobb angle outcomes in adolescents with idiopathic scoliosis - an assessor and statistician blinded randomized controlled trial. PLoS One. 2016;11(12): e0168746; doi: 10.1371/journal.pone.0168746.

14. Schreiber S, Parent EC, Moez EK, Hedden DM, Hill DL, Moreau M, et al. The effect of Schroth exercises added to the standard of care on the quality of life and muscle endurance in adolescents with idiopathic scoliosis - an assessor and statistician blinded randomized controlled trial: "SOSORT 2015 Award Winner". Scoliosis. 2015; 10:24; doi: 10.1186/s13013-015-0048-5.

15. Zapata KA, Wang-Price SS, Sucato DJ. Six-month follow-up of supervised spinal stabilization exercises for low back pain in adolescent idiopathic scoliosis. Pediatr Phys Ther. 2017;29(1):62-66; doi: 10.1097/PEP.00000 00000000325.

16. Farooqui SI, Siddiqui PQR, Ansari B, Farhad A. Effects of spinal mobilization techniques in the management of adolescent idiopathic scoliosis: a meta-analysis. Int J Health Sci. 2018;12(6):44-49.

17. Zapata KA, Sucato DJ, Jo CH. Physical therapy scoliosisspecific exercises may reduce curve progression in mild adolescent idiopathic scoliosis curves. Pediatr Phys Ther. 2019;31(3):280-285; doi: 10.1097/PEP.000000000000 0621.

18. Gao C, Zheng Y, Fan C, Yang Y, He C, Wong M. Could the clinical effectiveness be improved under the integration of orthotic intervention and scoliosis-specific exercise in managing adolescent idiopathic scoliosis? A randomized controlled trial study. Am J Phys Med Rehabil. 2019; 98(8):642-648; doi: 10.1097/phm.0000000000001160.

19. Mooney V, Gulick J, Pozos R. A preliminary report on the effect of measured strength training in adolescent idio- pathic scoliosis. J Spinal Disord. 2000;13(2):102-107; doi: 10.1097/00002517-200004000-00002.

20. Mclntire KL, Asher MA, Burton DC, Liu W. Treatment of adolescent idiopathic scoliosis with quantified trunk rotational strength training: a pilot study. J Spinal Disord Tech. 2008;21(5):349-358; doi: 10.1097/BSD.0b013e $318145 \mathrm{~b} 7 \mathrm{e}$.

21. Zheng Y, Dang Y, Yang Y, Li H, Zhang L, Lou EHM, et al. Whether orthotic management and exercise are equally effective to the patients with adolescent idiopathic scoliosis in mainland China? A randomized controlled trial study. Spine. 2018;43(9):494-503; doi: 10.1097/BRS. 0000000000002412.

22. Gomez JA, Hresko MT, Glotzbecker MP. Nonsurgical management of adolescent idiopathic scoliosis. J Am Acad Orthop Surg. 2016;24(8):555-564; doi: 10.5435/ JAAOS-D-14-00416.

23. Romano M, Minozzi S, Zaina F, Bettany-Saltikov J, Chockalingam N, Kotwicki T, et al. Exercises for adolescent idiopathic scoliosis: a Cochrane systematic review. Spine. 2013;38(14):E883-E893; doi: 10.1097/BRS.0b $013 e 31829459 f 8$.

24. Anwer S, Alghadir A, Abu Shaphe M, Anwar D. Effects of exercise on spinal deformities and quality of life in patients with adolescent idiopathic scoliosis. Biomed Res Int. 2015;2015:123848; doi: 10.1155/2015/123848.

25. Yagci G, Ayhan C, Yakut Y. Effectiveness of basic body awareness therapy in adolescents with idiopathic scoliosis: a randomized controlled study. J Back Musculoskelet Rehabil. 2018;31(4):693-701; doi: 10.3233/BMR170868.

26. Gür G, Ayhan C, Yakut Y. The effectiveness of core stabilization exercise in adolescent idiopathic scoliosis: a randomized controlled trial. Prosthet Orthot Int. 2017;41(3): 303-310; doi: 10.1177/0309364616664151. 